\title{
Application of Iterative Techniques for Electromagnetic Scattering From Dielectric Random and Reentrant Rough Surfaces
}

\author{
K. İnan and V. B. Ertürk, Member, IEEE
}

\begin{abstract}
Stationary [e.g., forward-backward method (FBM)] and nonstationary [e.g., conjugate gradient squared, quasi-minimal residual, and biconjugate gradient stabilized (Bi-CGSTAB)] iterative techniques are applied to the solution of electromagnetic wave scattering from dielectric random rough surfaces with arbitrary complex dielectric constants. The convergence issues as well as the efficiency and accuracy of all the approaches considered in this paper are investigated by comparing obtained scattering (in the form of normalized radar cross section) and surface field values with the numerically exact solution, computed by employing the conventional method of moments. It has been observed that similar to perfectly and imperfectly conducting rough surface cases, the stationary iterative FBM converges faster when applied to geometries yielding best conditioned systems but exhibits convergence difficulties for general geometries due to its inherit limitations. However, nonstationary techniques are, in general, more robust when applied to arbitrarily general dielectric random rough surfaces, which yield more ill-conditioned systems. Therefore, they might prove to be more suitable for general scattering problems. Besides, as opposed to the perfectly and imperfectly conducting rough surface cases, the Bi-CGSTAB method and FBM show two interesting behaviors for dielectric rough surface profiles: 1) FBM generally converges for reentrant surfaces when the vertical polarization is considered and 2) the Bi-CGSTAB method has a peculiar convergence problem for horizontal polarization. Unlike the other nonstationary iterative techniques used in this paper, where a Jacobi preconditioner is used, convergent results are obtained by using a block-diagonal preconditioner.
\end{abstract}

Index Terms-Iterative techniques, method of moments (MoM), normalized radar cross section (NRCS), random rough surface scattering.

\section{INTRODUCTION}

A CCURATE and efficient evaluation of scattering from rough surfaces [1], [2] has great importance for military and commercial applications. Therefore, various integral equation (IE)-based methods have been proposed for perfectly [3], [4]-[14] and imperfectly [15], [16] conducting as well as dielectric [17]-[23] random rough surfaces. However, the order of $N^{3}$ [i.e., $O\left(N^{3}\right)$ ], where $N$ is the number of un-

Manuscript received January 7, 2006; revised April 24, 2006.

K. İnan was with the Department of Electrical and Electronics Engineering, Bilkent University, 06800 Ankara, Turkey. He is now with the Electronic Packaging Laboratory, Department of Electronic Systems, Arizona State University, Mesa, AZ 85212 USA.

V. B. Ertürk is with the Department of Electrical and Electronics Engineering, Bilkent University, 06800 Ankara, Turkey (e-mail: vakur@ee.bilkent. edu.tr).

Digital Object Identifier 10.1109/TGRS.2006.878440 knowns, computation cost for the direct solution procedure of the resultant system of linear algebraic equations has led to the use of iterative methods for electrically large rough surfaces [6]-[16], [19]-[23]. Among them, the stationary forwardbackward method (FBM) has been widely used for both perfectly and imperfectly conducting rough surfaces and further accelerated using the spectral acceleration (SA) algorithm [14]. However, due to its stationary nature, FBM fails when the surface profile of interest is a multivalued one, such as the reentrant surface of a ship. Therefore, besides the generalized version of FBM, namely generalized FBM (GFBM) [12], various nonstationary algorithms have been proposed [3], [15], [16] because they prove to be much more robust in many relatively ill-conditioned situations where FBM fails [3], [15].

On the other hand, when the scattering of electromagnetic waves from dielectric random rough surfaces is considered, the solution procedure for majority of the IE-based studies is restricted to the conventional method of moments (MoM) [21], which suffers from the excess computational cost requirements when applied to electrically large profiles. Investigation of these cases is very important since perfectly or highly conducting surfaces [i.e., use of impedance boundary conditions (IBC)] cannot model many dielectric-type surfaces such as soil surfaces at microwave frequencies [23]. Therefore, recently, the original FBM has been modified (called modified FBM) to handle scattering from dielectric one-dimensional (1-D) profiles with arbitrary complex dielectric constant [22] and then applied to scattering problems from natural soils modeled by dielectric fractal profiles [23]. However, similar to the perfectly and imperfectly conducting cases, modified FBM has some inherent convergence limitations due to its stationary nature when applied to certain dielectric random rough surfaces that are relatively rough and less correlated. Furthermore, the modified FBM may fail in the case of a reentrant dielectric rough surface (random/deterministic).

Considering these facts in mind, in this paper, the work presented in [3] has been extended to dielectric random and dielectric reentrant rough surfaces. Modified FBM as the stationary and several nonstationary iterative techniques are applied to the solution of electromagnetic wave scattering from dielectric rough surfaces. Both random dielectric profiles, which are described by Gaussian processes with Gaussian and exponential correlations and deterministic reentrant dielectric profiles, are considered. The convergence issues as well as the efficiency and accuracy of all the approaches are investigated by comparing obtained scattering and surface field results with the numerically exact solution, computed by employing the conventional MoM. Among the nonstationary techniques, emphasis is given 
to conjugate gradient squared (CGS), quasi-minimal residual (QMR), and biconjugate gradient stabilized (Bi-CGSTAB) [24] since the others possess a much slower convergence rate for the dielectric rough surface profiles (random/deterministic) considered in this paper. It has been observed that when the considered profile (random or deterministic) yields a relatively well-conditioned moment-method interaction matrix, application of modified FBM gives the fastest convergence. However, many arbitrary profiles yield more ill-conditioned systems, which are problematic for the modified FBM due to its inherent limitations. Thus, nonstationary techniques are implemented to such profiles because, in general, they are more robust. Very accurate and reasonably efficient results are obtained for both random and deterministic rough surface profiles, in particular by using CGS. Besides, two interesting behaviors of FBM and the Bi-CGSTAB method have been observed for dielectric rough surfaces. First, FBM, which is known to be problematic for perfectly and imperfectly conducting reentrant surfaces, may converge for dielectric reentrant surfaces when the incident electric field is vertically polarized (i.e., VV polarization). Second, the Bi-CGSTAB method is known to work well for perfectly and imperfectly conducting surfaces. However, for dielectric surfaces, a carefully chosen preconditioner (in this case, we used a block-diagonal preconditioner) may be required for its convergence when the incident electric field is horizontally polarized (i.e., $\mathrm{HH}$ polarization).

This paper is organized as follows. In Section II, the geometry and the formulation of the problem are given. Section III is composed of numerical results and their discussions. Both farzone scattered and surface fields are evaluated using iterative techniques whenever they converge and compared with the conventional MoM solution. Accuracy, efficiency, and convergence properties of both stationary and nonstationary iterative techniques used in this paper are explored. An $e^{j w t}$ time convention is employed and suppressed throughout this paper.

\section{FORMULATION}

Consider the 1-D rough surface profile embedded in a twodimensional (2-D) space as illustrated in Fig. 1. Surface height profile and electromagnetic fields are assumed to be constant along the $y$ direction [21]. For this geometry, if the incident field is horizontally polarized, then the surface electric and magnetic fields can be evaluated solving the following pair of IEs [17], [21]-[23]:

$$
\begin{aligned}
E^{\mathrm{inc}}(\mathbf{r})=\frac{E(\mathbf{r})}{2}+\int_{l}\left\{j \omega \mu_{0} \phi_{0}\left(\mathbf{r}, \mathbf{r}^{\prime}\right) J_{s}\left(\mathbf{r}^{\prime}\right)\right. \\
\left.+E\left(\mathbf{r}^{\prime}\right)\left[\hat{n}^{\prime} \cdot \nabla \phi_{0}\left(\mathbf{r}, \mathbf{r}^{\prime}\right)\right]\right\} d l^{\prime} \\
0=\frac{E(\mathbf{r})}{2}-\int_{l}\left\{j \omega \mu_{0} \phi_{1}\left(\mathbf{r}, \mathbf{r}^{\prime}\right) J_{s}\left(\mathbf{r}^{\prime}\right)\right. \\
\left.+E\left(\mathbf{r}^{\prime}\right)\left[\hat{n}^{\prime} \cdot \nabla \phi_{1}\left(\mathbf{r}, \mathbf{r}^{\prime}\right)\right]\right\} d l^{\prime}
\end{aligned}
$$

where $\mathbf{E}^{\text {inc }}=E^{\text {inc }} \hat{y}$ and $\mathbf{E}=E \hat{y}$ are incident and total electric fields, respectively. $\mathbf{J}_{s}=\hat{n} \times \mathbf{H}=J_{s} \hat{y}$ is the equivalent electric surface current density, and $\hat{n}$ is the outgoing normal to the surface. On the other hand, if the incident field is vertically

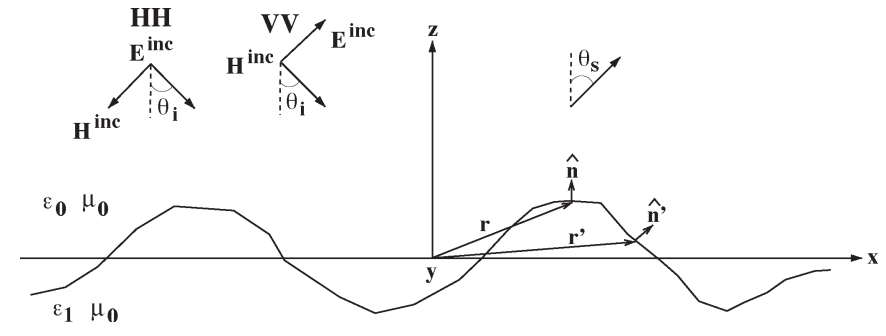

Fig. 1. Geometry of scattering from dielectric random rough surface problem.

polarized, then the surface electric and magnetic fields can be evaluated by solving the following pair of IEs [21]-[23]:

$$
\begin{aligned}
H^{\mathrm{inc}}(\mathbf{r})=\frac{H(\mathbf{r})}{2}+\int_{l}\left\{j \omega \epsilon_{0} \phi_{0}\left(\mathbf{r}, \mathbf{r}^{\prime}\right) K_{s}\left(\mathbf{r}^{\prime}\right)\right. \\
\left.+H\left(\mathbf{r}^{\prime}\right)\left[\hat{n}^{\prime} \cdot \nabla \phi_{0}\left(\mathbf{r}, \mathbf{r}^{\prime}\right)\right]\right\} d l^{\prime} \\
0=\frac{H(\mathbf{r})}{2}-\int_{l}\left\{j \omega \epsilon_{1} \phi_{1}\left(\mathbf{r}, \mathbf{r}^{\prime}\right) K_{s}\left(\mathbf{r}^{\prime}\right)\right. \\
\left.+H\left(\mathbf{r}^{\prime}\right)\left[\hat{n}^{\prime} \cdot \nabla \phi_{1}\left(\mathbf{r}, \mathbf{r}^{\prime}\right)\right]\right\} d l^{\prime}
\end{aligned}
$$

where $\mathbf{H}^{\text {inc }}=H^{\text {inc }} \hat{y}$ and $\mathbf{H}=H \hat{y}$ are incident and total magnetic fields, respectively. $\mathbf{K}_{s}=-\hat{n} \times \mathbf{E}=K_{s} \hat{y}$ is the equivalent magnetic surface current density, and $\epsilon_{0}, \epsilon_{1}=\epsilon_{r} \epsilon_{0}$ are the dielectric constants of the upper and lower media, respectively. In (1)-(4), both $\mathbf{r}$ and $\mathbf{r}^{\prime}$ belong to the surface profile, and

$$
\phi_{0,1}\left(\mathbf{r}, \mathbf{r}^{\prime}\right)=-\frac{j}{4} H_{0}^{(2)}\left(k_{0,1}\left|\mathbf{r}-\mathbf{r}^{\prime}\right|\right)
$$

is the 2-D free-space Green's function (zeroth-order Hankel function of the second kind), where $k_{0}, k_{1}$ are the propagation constants of the upper and lower media, respectively. Equations (1)-(4) assume the lower space to be homogeneous, unlimited, and with the same magnetic permeability, i.e., $\mu_{0}$, of the upper one.

In this paper, the matrix formulation is given for (1)-(2). However, the same procedure applies for (3)-(4). Similar to [21], using rectangular pulse basis functions and performing a point matching method, the IE pair (1)-(2) can be converted into a pair of matrix equations for the unknowns $\underline{E}$ and $\underline{J_{s}}$ given by

$$
\left[\begin{array}{ll}
\underline{\underline{S}}_{0} & \underline{\underline{Z}}_{0} \\
\underline{\underline{S}}_{1} & \underline{\underline{Z}}_{1}
\end{array}\right]\left[\begin{array}{l}
\underline{E} \\
\underline{J}_{s}
\end{array}\right]=\left[\begin{array}{l}
\underline{E}^{\mathrm{inc}} \\
\underline{0}
\end{array}\right]
$$

where the size of the matrix is $2 N \times 2 N$, with $N$ being the number of rectangular pulse basis functions used to expand the unknown current density $J_{s}$ and the unknown surface field $E$ over the entire illuminated surface contour. The full expressions for the coefficients of the matrix elements are given in [21], and for the sake of completeness, they are repeated at the Appendix. The matrix equation given by (6) is solved for the unknown surface fields by first employing a direct solution method (LU decomposition) to obtain a numerically exact reference solution. Then, the accuracy, efficiency, and convergence properties of both stationary and nonstationary iterative techniques are investigated by implementing them 
(a)

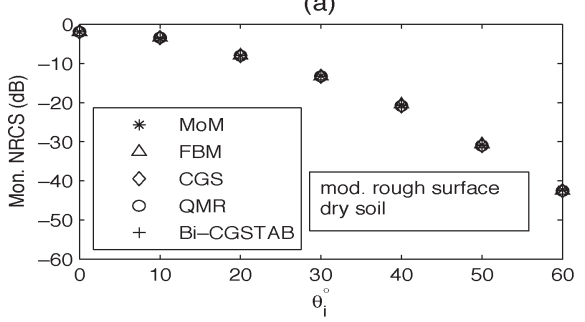

(c)

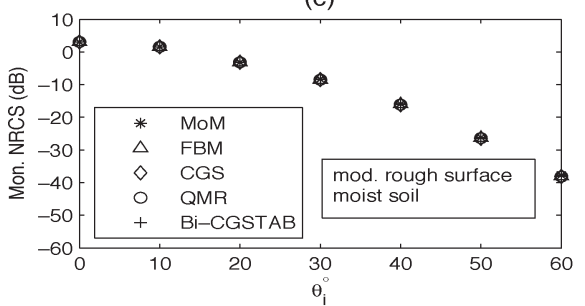

(e)

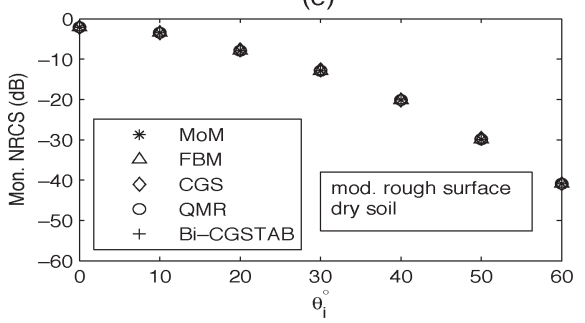

(g)

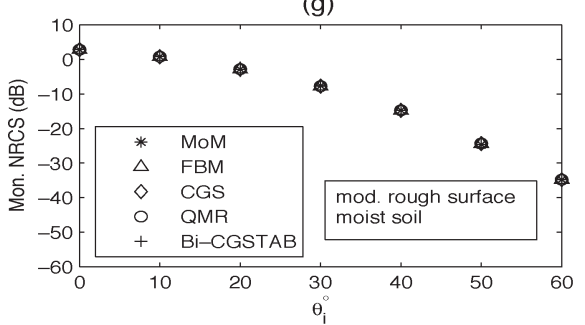

(b)

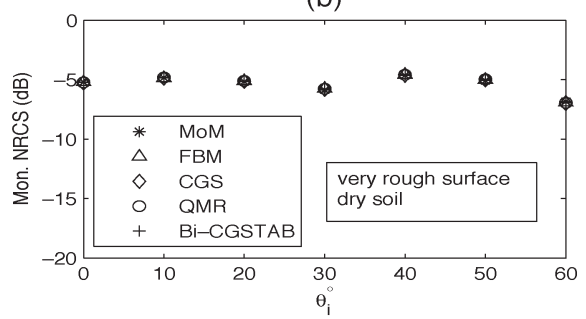

(d)

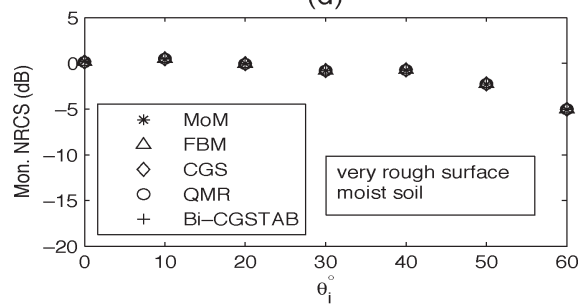

(f)

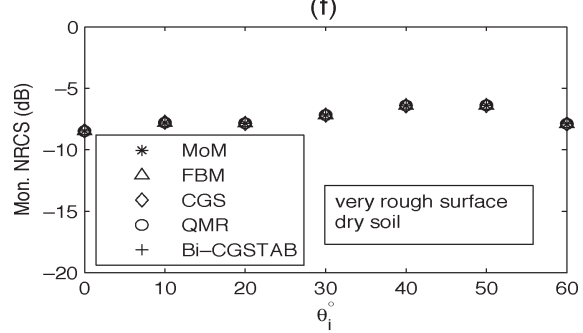

(h)

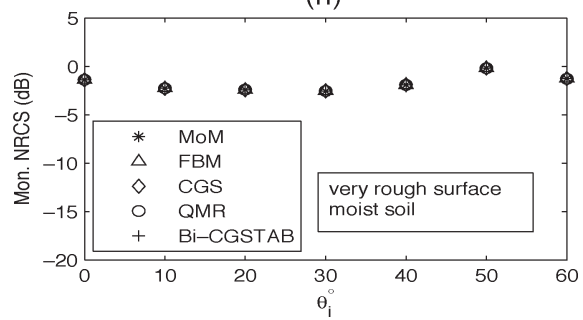

Fig. 2. Comparison of the monostatic NRCS values of the iterative methods with the conventional MoM results, for various angles of incidence at 1 GHz. (a) $\mathrm{HH}$ polarization, $\sigma=\lambda / 6$, rms slope $=13^{\circ}$ (i.e., moderately rough surface), and $\epsilon_{r}=4$ (i.e., dry soil). (b) $\mathrm{HH}$ polarization, $\sigma=0.707 \lambda$, rms slope $=45^{\circ}$ (i.e., very rough surface), and $\epsilon_{r}=4$. (c) $\mathrm{HH}$ polarization, $\sigma=\lambda / 6$, rms slope $=13^{\circ}$, and $\epsilon_{r}=15-j 4$ (i.e., moist soil). (d) $\mathrm{HH}$ polarization, $\sigma=0.707 \lambda$, rms slope $=45^{\circ}$, and $\epsilon_{r}=15-j 4$. (e) VV polarization, $\sigma=\lambda / 6$, rms slope $=13^{\circ}$, and $\epsilon_{r}=4$. (f) VV polarization, $\sigma=0.707 \lambda$, rms slope $=45^{\circ}$, and $\epsilon_{r}=4$. (g) VV polarization, $\sigma=\lambda / 6$, rms slope $=13^{\circ}$, and $\epsilon_{r}=15-j 4$. (h) VV polarization, $\sigma=0.707 \lambda$, rms slope $=45^{\circ}$, and $\epsilon_{r}=15-j 4$.

to (6) for the dielectric rough surface problem. Therefore, modified FBM [22], [23] as the stationary and several nonstationary iterative techniques [24] are implemented. The nonstationary algorithms are members of the general conjugate direction class of algorithms developed for asymmetric/nonHermitian matrices [3], whose pseudocodes are given in [24]. Among them, emphasis is given to the CGS, QMR, and BiCGSTAB methods since much quicker convergence rates have been obtained by using them for the dielectric rough surface profiles (both random and deterministic) considered in this paper. All nonstationary techniques are applied directly to (6) without any modification. A Jacobi preconditioner was initially used for all of them as discussed in [24]. However, unlike perfectly and imperfectly conducting rough surface cases, the Bi-CGSTAB method exhibited a peculiar convergence problem when dielectric rough surface profiles are considered for the $\mathrm{HH}$ polarization. Therefore, a block-diagonal preconditioner is used. In this preconditioner, blocks are chosen to be $2 \times$ 2 submatrices, whose details are given in [25]. Although Bi-
CGSTAB results converge with this preconditioner, the CPU time for each iteration slightly increases compared with other cases where the Jacobi preconditioner is used.

Once the surface fields are evaluated, the far zone scattered field for $z>0$ can be calculated. For the $\mathrm{HH}$ polarization case, it is given by [26]

$$
\begin{gathered}
E^{s}(\mathbf{r})=\frac{k_{0} \exp \left(j \frac{\pi}{4}\right)}{\sqrt{8 \pi k_{0} r}} \exp \left(-j k_{0} r\right) \int_{l}\left[\eta J_{s}\left(\mathbf{r}^{\prime}\right)-\left(\hat{n}^{\prime} \cdot \hat{n}_{s}\right) E\left(\mathbf{r}^{\prime}\right)\right] \\
\quad \times \exp \left(j k_{0} \hat{n}_{s} \cdot \mathbf{r}^{\prime}\right) \sqrt{1+(d z / d x)^{2}} g\left(x^{\prime}\right) d x^{\prime}
\end{gathered}
$$

For the VV polarization case, we use

$$
\begin{gathered}
H^{s}(\mathbf{r})=\frac{k_{0} \exp \left(j \frac{\pi}{4}\right)}{\sqrt{8 \pi k_{0} r}} \exp \left(-j k_{0} r\right) \int_{l}\left[\frac{K_{s}\left(\mathbf{r}^{\prime}\right)}{\eta}-\left(\hat{n}^{\prime} \cdot \hat{n}_{s}\right) H\left(\mathbf{r}^{\prime}\right)\right] \\
\quad \times \exp \left(j k_{0} \hat{n}_{s} \cdot \mathbf{r}^{\prime}\right) \sqrt{1+(d z / d x)^{2}} g\left(x^{\prime}\right) d x^{\prime} .
\end{gathered}
$$


(a)

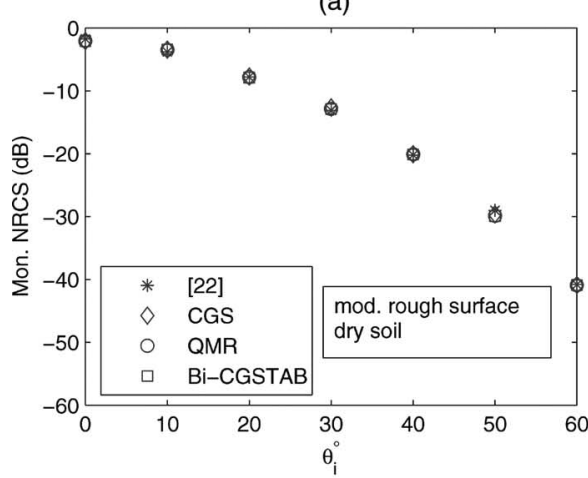

(c)

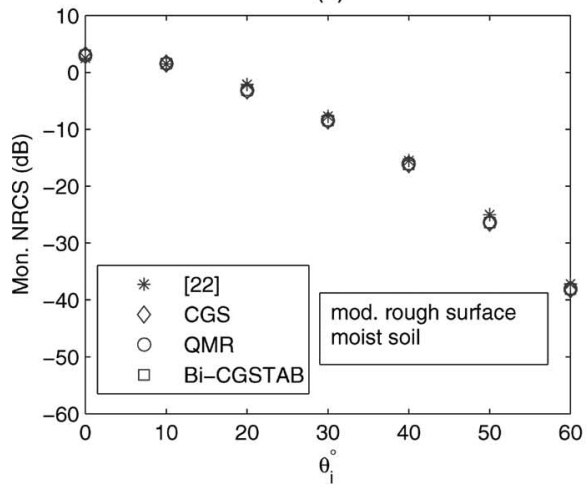

(b)

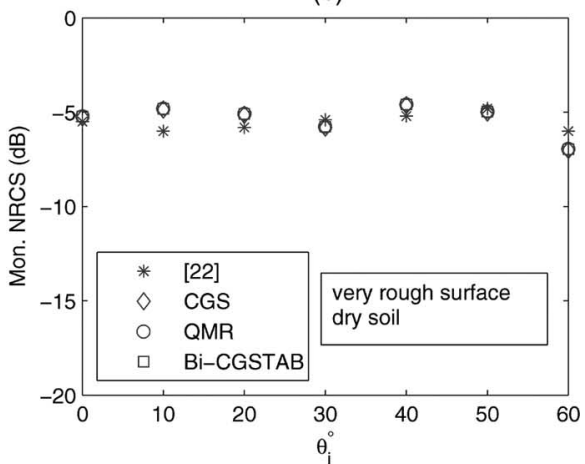

(d)

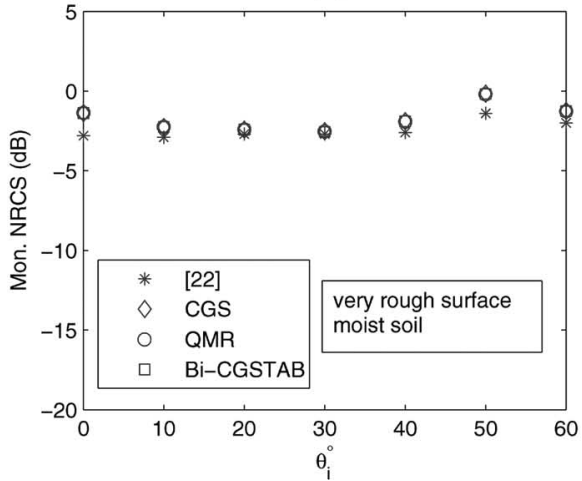

Fig. 3. Comparison of the monostatic NRCS values from [22] for various angles of incidence with the iterative results obtained in this paper at $1 \mathrm{GHz}$. (a) VV polarization, $\sigma=\lambda / 6$, rms slope $=13^{\circ}$, and $\epsilon_{r}=4$. (b) HH polarization, $\sigma=0.707 \lambda$, rms slope $=45^{\circ}$, and $\epsilon_{r}=4$. (c) HH polarization, $\sigma=\lambda / 6$, rms slope $=13^{\circ}$, and $\epsilon_{r}=15-j 4$. (d) $\mathrm{VV}$ polarization, $\sigma=0.707 \lambda$, rms slope $=45^{\circ}$, and $\epsilon_{r}=15-j 4$.

In (7) and (8), $\hat{n}_{s}$ is the unit vector indicating the scattering direction, $\mathbf{r}$ is the point in the far zone, $\mathbf{r}^{\prime}$ belongs to the surface profile, $\eta$ is the free-space intrinsic impedance, and $g(\cdot)$ is the (slowly varying) illumination tapering function that reduces the edge effect and is assumed to be negligible for $\left|x^{\prime}\right| \geq L_{s} / 2$, where $L_{s}$ is the profile length [21]. More precisely, we assume that $g(x)$ is Gaussian with $g(0)=1$ and a width such that $g\left(L_{s} / 2\right)=10^{-6}$, so that the profile effective length can be defined as

$$
L_{\mathrm{eff}}=\int_{L_{s}} g^{2}\left(x^{\prime}\right) d x^{\prime}
$$

which is directly related to the profile length $L_{s}$ [17], [21].

Finally, the noncoherent normalized radar cross section (NRCS) of a 1-D profile for the $\mathrm{HH}$ polarization is expressed as [22], [27]

$$
\sigma^{0}=\frac{2 \pi r\left(\left\langle\left|E^{s}\right|^{2}\right\rangle-\left|\left\langle E^{s}\right\rangle\right|^{2}\right)}{L_{\mathrm{eff}}\left|E^{\text {inc }}\right|^{2}}
$$

where $\langle\cdot\rangle$ stands for the mean value. This definition is based on the hypothesis that the mean square value of the scattered field is directly proportional to the profile effective length $L_{\text {eff }}$ and has no other dependence on the illuminating function shape, so that the NRCS turns out to depend only on surface properties [21]. For the VV polarization, $E^{s}$ and $E^{\text {inc }}$ should be replaced by $H^{s}$ and $H^{\text {inc }}$, respectively.

\section{NumERICAL RESUlTS AND Discussions}

\section{A. Accuracy of the Iterative Methods}

To assess the accuracy of the iterative algorithms proposed in this paper for the matrix equation given by (6), the noncoherent NRCS results evaluated using (10) are obtained and compared with: 1) the ones computed via the conventional MoM and 2) the ones given in [22]. The comparison methodology is similar to the one used in [22]. Briefly, 160 independent sample profiles with prescribed statistics are generated. Then, fields scattered by different surfaces are evaluated using the conventional MoM and stationary and nonstationary iterative techniques and are subsequently averaged to compute the NRCS. Consequently, the error estimate of $\sigma^{0}$ is less than $1 \mathrm{~dB}$ with a probability of 0.997 [22]. In all results, unless specified, the width of each basis function is $\lambda / 10$, the frequency is $1 \mathrm{GHz}$ $(\lambda=0.3 \mathrm{~m})$, and the length of the surfaces is $15 \mathrm{~m}$.

In Fig. 2, the monostatic HH and VV NRCS of the moderately rough and very rough Gaussian correlated surfaces, evaluated by MoM and iterative techniques (i.e., FBM, CGS, QMR, Bi-CGSTAB), are plotted as a function of the angle of incidence $\theta_{i}$. Both the dry soil (i.e., $\epsilon_{r}=4$ ) and moist soil (i.e., $\epsilon_{r}=15-j 4$ ) cases are considered. For the moderately rough surface, $\sigma$ is set to $\lambda / 6$ and the root mean square (rms) slope is set to $13^{\circ}$, whereas for the very rough surface, these parameters are set to $0.707 \lambda$ and $45^{\circ}$, respectively. These results match very well with the ones given in [22], as shown in Fig. 3, where the monostatic NRCS results obtained via CGS, QMR, and Bi-CGSTAB (FBM is the same) are compared with those given in [22] for some cases including both the $\mathrm{HH}$ and $\mathrm{VV}$ 
(a)

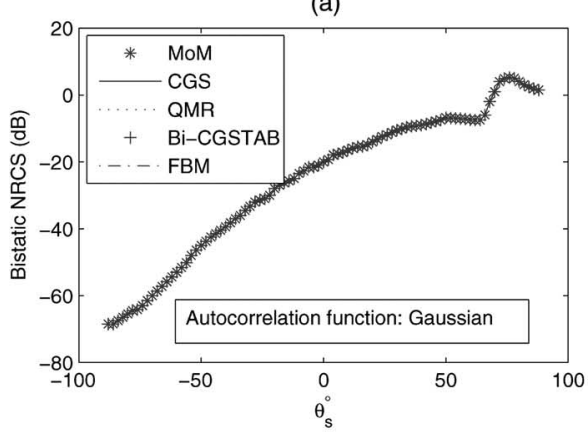

(c)

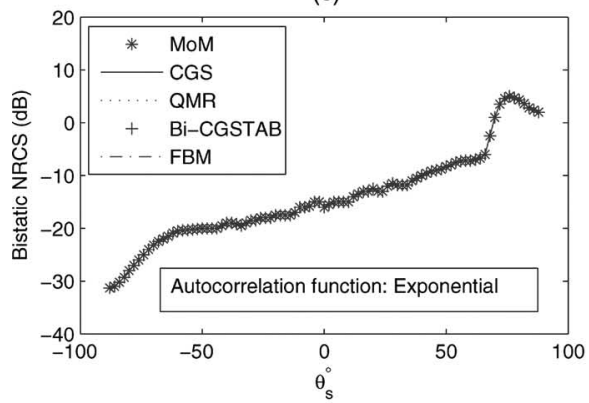

(e)

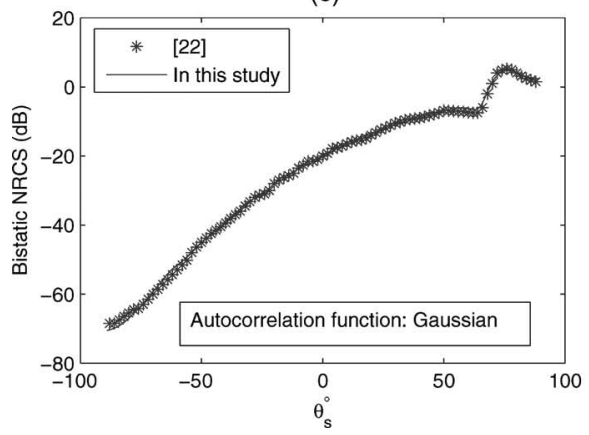

(b)

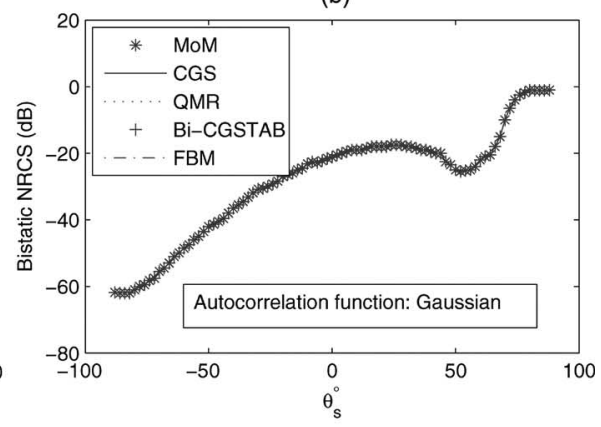

(d)

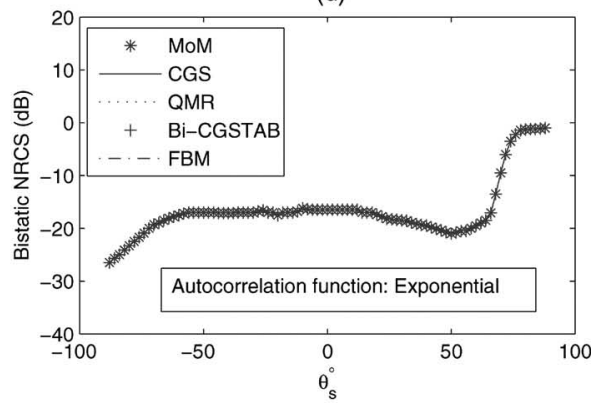

(f)

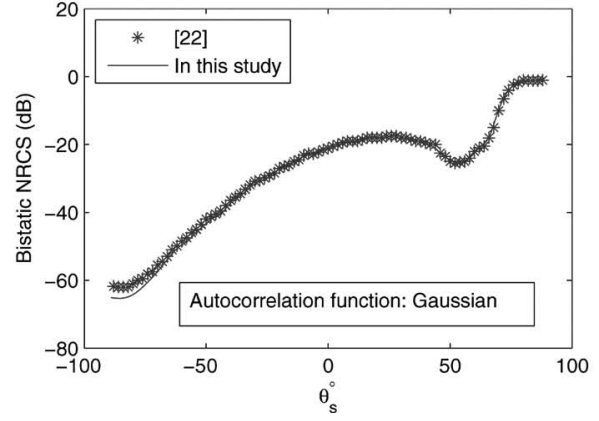

Fig. 4. Comparison of the bistatic NRCS values obtained from the iterative methods (a)-(d) with the conventional MoM solution and (e)-(f) with the result given in [22] for both $\mathrm{HH}$ and VV polarizations. Dielectric constant is $\epsilon_{r}=4$. Frequency is $1 \mathrm{GHz} . \sigma=\lambda / 6$, and rms slope $=13^{\circ}$. (a) HH polarization for a Gaussian correlated profile. (b) VV polarization for a Gaussian correlated profile. (c) HH polarization for an exponential correlated profile. (d) VV polarization for an exponential correlated profile. (e) HH polarization for a Gaussian correlated profile. (f) VV polarization for a Gaussian correlated profile.

polarizations for the same parameters. Consequently, based on Figs. 2 and 3, very accurate results are obtained. The difference between the results in this paper and the ones given in [22] is smaller than $1 \mathrm{~dB}$ for all cases. Furthermore, the rms error [22, eq. (23)] between the iterative methods and the MoM is found to be smaller than $0.05 \mathrm{~dB}$, which shows the accuracy of the stopping criterion and the good estimates of the surface NRCS by iterative algorithms.

Similar to the monostatic NRCS comparisons, bistatic NRCS comparisons have been performed. Fig. 4(a) and (b) depicts the bistatic $\mathrm{HH}$ and VV NRCS of a moderately rough Gaussian correlated surface versus scattering angle $\theta_{s}$, respectively. Results obtained from the iterative techniques are compared with the MoM result for a dielectric constant of $\epsilon_{r}=4$ (i.e., dry soil). In Fig. 4(c) and (d), a similar result is also given for an exponentially correlated rough surface profile where all the parameters are kept same. In Fig. 4(e) and (f), results of bistatic $\mathrm{HH}$ and VV NRCS derived in this paper for a moderately rough Gaussian correlated surface are compared with the ones given in [22]. As shown in Fig. 4, the agreement between the results obtained from the iterative techniques and the MoM result, and the agreement between the results in this paper and the ones given in [22] are all very good. Consequently, establishing a full confidence in our results, we can investigate their efficiency and test them for electromagnetic scattering in more general dielectric rough surface profiles.

\section{B. Efficiency and Convergence Rate of the Nonstationary Iterative Methods}

Efficiencies and convergence rates of the nonstationary iterative methods considered in this paper, namely CGS, QMR, and Bi-CGSTAB, are investigated in a similar fashion to [22] in which the FBM has been discussed. For the CGS, QMR, and Bi-CGSTAB methods, Tables I-IV from [22] are reproduced (see Tables I-IV). Results obtained by using FBM are similar to [22] and, hence, are not repeated here. Following the same methodology explained in [22], for each table, the minimum number of iterations $n_{0}$ is given such that an error of $r\left(n_{0}\right)<10^{-2}$ is satisfied for both Gaussian and exponentially correlated autocorrelation surfaces with different values of standard deviation $\sigma$ and correlation length $L_{c}$. Each value in all tables is obtained by averaging over ten surface realizations, and "nc" means there is no convergence (i.e., the error criterion 
TABLE I

Average Minimum Number of Iterations $n_{0}$ Such That $r\left(n_{0}\right)<$ $10^{-2}$ IS SATISFIED FOR DiFFERENT VALUES OF STANDARD DEVIATION $\sigma$ AND CORRELATION LENGTH $L_{c}$ AND FOR VV AND HH POLARIZATIONS. ANGLE OF INCIDENCE $=60^{\circ}$. SURFACE AUTOCORRELATION FUNCTION: Gaussian. Relative Dielectric Constant: $\epsilon_{r}=4$

\begin{tabular}{||c|c|c|c|c|c|c|c||}
\hline $\begin{array}{c}\text { Iterative } \\
\text { Method }\end{array}$ & $\begin{array}{c}\sigma / \lambda \\
\sigma / L_{c}\end{array}$ & $\begin{array}{c}0.1 \\
\text { VV-HH }\end{array}$ & $\begin{array}{c}0.3 \\
\text { VV-HH }\end{array}$ & $\begin{array}{c}0.5 \\
\text { VV-HH }\end{array}$ & $\begin{array}{c}0.7 \\
\text { VV-HH }\end{array}$ & $\begin{array}{c}1.0 \\
\text { VV-HH }\end{array}$ & $\begin{array}{c}10.0 \\
\text { VV-HH }\end{array}$ \\
\hline \multirow{4}{*}{ CGS } & 0.15 & $29-31$ & $28-29$ & $27-28$ & $26-31$ & $25-25$ & $25-29$ \\
\cline { 2 - 8 } & 0.20 & $32-35$ & $33-32$ & $27-28$ & $23-30$ & $21-29$ & $22-32$ \\
\cline { 2 - 8 } & 0.33 & $31-33$ & $36-36$ & $28-36$ & $24-32$ & $22-28$ & $23-29$ \\
\cline { 2 - 8 } & 0.71 & $33-31$ & $39-42$ & $28-41$ & $26-33$ & $23-33$ & $24-31$ \\
\cline { 2 - 8 } & 1.00 & $33-34$ & $42-50$ & $30-47$ & $26-42$ & $23-35$ & $24-33$ \\
\hline Bi-CGSTAB & 0.15 & $24-24$ & $21-25$ & $30-35$ & $26-26$ & $19-24$ & $14-23$ \\
\cline { 2 - 8 } & 0.20 & $29-28$ & $32-43$ & $34-48$ & $28-24$ & $22-23$ & $19-23$ \\
\cline { 2 - 8 } & 0.33 & $35-35$ & $48-54$ & $38-53$ & $41-33$ & $27-33$ & $21-22$ \\
\cline { 2 - 8 } & 0.71 & $41-37$ & $61-68$ & $42-68$ & $43-42$ & $32-35$ & $25-25$ \\
\cline { 2 - 8 } & 1.00 & $45-50$ & $78-76$ & $58-75$ & $48-73$ & $41-54$ & $27-30$ \\
\hline \multirow{5}{*}{ QMR } & 0.15 & $43-46$ & $46-42$ & $43-42$ & $41-41$ & $44-39$ & $43-53$ \\
\cline { 2 - 8 } & 0.20 & $47-40$ & $53-37$ & $48-41$ & $47-40$ & $46-36$ & $45-48$ \\
\cline { 2 - 8 } & 0.33 & $51-46$ & $62-43$ & $51-67$ & $48-48$ & $46-43$ & $45-51$ \\
\cline { 2 - 7 } & 0.71 & $58-56$ & $71-49$ & $59-71$ & $55-56$ & $51-48$ & $46-47$ \\
\cline { 2 - 7 } & 1.00 & $69-65$ & $80-64$ & $67-84$ & $69-62$ & $57-68$ & $48-50$ \\
\hline
\end{tabular}

TABLE II

Average Minimum Number of Iterations $n_{0}$ Such That $r\left(n_{0}\right)<$ $10^{-2}$ IS SATISFIED FOR DiFFERENT VALUES OF STANDARD DEVIATION $\sigma$ AND CORRELATION LENGTH $L_{c}$ AND FOR VV AND HH POLARIZATIONS. ANGLE OF INCIDENCE $=60^{\circ}$. SURFACE AUTOCORRELATION FUNCTION: EXPONENTIAL. Relative Dielectric CONSTANT: $\epsilon_{r}=4$

\begin{tabular}{||c|c|c|c|c|c|c|c||}
\hline $\begin{array}{c}\text { Iterative } \\
\text { Method }\end{array}$ & $\begin{array}{c}\sigma / \lambda \\
\sigma / L_{c}\end{array}$ & $\begin{array}{c}0.1 \\
\text { VV-HH }\end{array}$ & $\begin{array}{c}0.3 \\
\text { VV-HH }\end{array}$ & $\begin{array}{c}0.5 \\
\text { VV-HH }\end{array}$ & $\begin{array}{c}0.7 \\
\text { VV-HH }\end{array}$ & $\begin{array}{c}1.0 \\
\text { VV-HH }\end{array}$ & $\begin{array}{c}10.0 \\
\text { VV-HH }\end{array}$ \\
\hline \multirow{4}{*}{ CGS } & 0.15 & $24-24$ & $22-26$ & $21-34$ & $25-37$ & $31-48$ & nc-nc \\
\cline { 2 - 8 } & 0.20 & $26-25$ & $28-39$ & $32-44$ & $31-42$ & $\mathbf{3 6 - 5 1}$ & nc-nc \\
\cline { 2 - 8 } & 0.33 & $30-31$ & $34-45$ & $43-51$ & $\mathbf{4 1 - 5 0}$ & $\mathbf{4 3 - 5 6}$ & nc-nc \\
\cline { 2 - 8 } & 0.71 & $31-33$ & $42-45$ & $\mathbf{5 1 - 6 9}$ & $\mathbf{5 7 - n c}$ & nc-nc & nc-nc \\
\cline { 2 - 8 } & 1.00 & $35-32$ & $\mathbf{5 5 - n c}$ & $\mathbf{6 8 - n c}$ & $\mathbf{8 2 - n c}$ & nc-nc & nc-nc \\
\hline Bi-CGSTAB & 0.15 & $28-33$ & $36-36$ & $43-44$ & $58-62$ & $63-58$ & nc-nc \\
\cline { 2 - 8 } & 0.20 & $30-31$ & $42-45$ & $51-48$ & $69-60$ & $\mathbf{6 8 - 8 0}$ & nc-nc \\
\cline { 2 - 8 } & 0.33 & $31-32$ & $41-42$ & $68-71$ & $\mathbf{8 2 - 1 0 2}$ & nc-nc & nc-nc \\
\cline { 2 - 8 } & 0.71 & $37-32$ & $76-74$ & nc-nc & nc-nc & nc-nc & nc-nc \\
\cline { 2 - 8 } & 1.00 & $43-35$ & $\mathbf{7 8 - 7 9}$ & nc-nc & nc-nc & nc-nc & nc-nc \\
\hline \multirow{5}{*}{ QMR } & 0.15 & $43-41$ & $48-52$ & $51-68$ & $62-80$ & $72-99$ & nc-nc \\
\cline { 2 - 8 } & 0.20 & $45-45$ & $63-69$ & $64-86$ & $73-100$ & $\mathbf{7 8 - 1 3 2}$ & nc-nc \\
\cline { 2 - 8 } & 0.33 & $45-47$ & $76-78$ & $76-81$ & $\mathbf{8 5 - 9 5}$ & $\mathbf{1 0 6 - n c}$ & nc-nc \\
\cline { 2 - 7 } & 0.71 & $49-47$ & $85-88$ & $\mathbf{1 0 4 - 9 6}$ & $\mathbf{1 2 8 - n c}$ & nc-nc & nc-nc \\
\cline { 2 - 7 } & 1.00 & $53-51$ & $\mathbf{1 0 1 - n c}$ & $\mathbf{1 2 7 - n c}$ & nc-nc & nc-nc & nc-nc \\
\hline
\end{tabular}

defined above is not met) for at least one out of ten realizations. The boldfaced numbers indicate the $n_{0}$ values for a converged result, which has been reported as nc in [22], where FMB is used. In all tables, the $\sigma / \lambda$ ratio defines the surface roughness. An increase in this ratio corresponds to an increase in the roughness of the surface. Similarly, the $\sigma / L_{c}$ ratio defines the correlation between the samples. As this ratio increases, the correlation decreases. The surface autocorrelation function (Gaussian or exponential) and the relative dielectric constant $\epsilon_{r}$ used to generate the results are given at the top of each table. Finally, for all cases, the angle of incidence is set to $60^{\circ}$.

An investigation of the results tabulated in Tables I-IV and their comparison with the FBM results given in [22] show that FBM, as a stationary iterative method, has a much quicker
TABLE III

Average Minimum Number of Iterations $n_{0}$ Such That $r\left(n_{0}\right)<$ $10^{-2}$ Is SATISFIED For DifFERENT VALUES OF STANDARD DEVIATION $\sigma$ AND CORRELATION LENGTH $L_{c}$ AND FOR VV AND HH POLARIZATIONS. ANGLE OF INCIDENCE $=60^{\circ}$. SURFACE AUTOCORRELATION FUNCTION: Gaussian. Relative Dielectric Constant: $\epsilon_{r}=15-j 4$

\begin{tabular}{||c|c|c|c|c|c|c|c||}
\hline $\begin{array}{c}\text { Iterative } \\
\text { Method }\end{array}$ & $\begin{array}{c}\sigma / \lambda \\
\sigma / L_{c}\end{array}$ & $\begin{array}{c}0.1 \\
\text { VV-HH }\end{array}$ & $\begin{array}{c}0.3 \\
\text { VV-HH }\end{array}$ & $\begin{array}{c}0.5 \\
\text { VV-HH }\end{array}$ & $\begin{array}{c}0.7 \\
\text { VV-HH }\end{array}$ & $\begin{array}{c}1.0 \\
\text { VV-HH }\end{array}$ & $\begin{array}{c}10.0 \\
\text { VV-HH }\end{array}$ \\
\hline \multirow{4}{*}{ CGS } & 0.15 & $9-24$ & $8-25$ & $9-23$ & $9-23$ & $9-24$ & $7-27$ \\
\cline { 2 - 8 } & 0.20 & $11-23$ & $11-28$ & $12-25$ & $13-24$ & $11-22$ & $8-21$ \\
\cline { 2 - 8 } & 0.33 & $13-21$ & $14-33$ & $16-31$ & $16-27$ & $13-25$ & $8-24$ \\
\cline { 2 - 8 } & 0.71 & $15-30$ & $17-36$ & $19-35$ & $19-27$ & $18-25$ & $10-22$ \\
\cline { 2 - 8 } & 1.00 & $15-36$ & $24-48$ & $24-38$ & $22-29$ & $19-26$ & $11-22$ \\
\hline Bi-CGSTAB & 0.15 & $10-34$ & $12-36$ & $12-35$ & $11-40$ & $12-32$ & $10-35$ \\
\cline { 2 - 8 } & 0.20 & $14-35$ & $21-37$ & $22-38$ & $14-38$ & $16-29$ & $11-33$ \\
\cline { 2 - 8 } & 0.33 & $17-35$ & $23-41$ & $32-41$ & $18-39$ & $19-32$ & $14-26$ \\
\cline { 2 - 8 } & 0.71 & $21-31$ & $32-45$ & $48-44$ & $25-41$ & $25-30$ & $17-27$ \\
\cline { 2 - 8 } & 1.00 & $30-38$ & $46-51$ & $62-55$ & $28-39$ & $31-50$ & $19-25$ \\
\hline \multirow{5}{*}{ QMR } & 0.15 & $19-33$ & $19-35$ & $18-34$ & $17-36$ & $18-34$ & $16-46$ \\
\cline { 2 - 8 } & 0.20 & $21-33$ & $24-38$ & $22-39$ & $28-38$ & $24-36$ & $17-41$ \\
\cline { 2 - 8 } & 0.33 & $23-35$ & $29-41$ & $27-44$ & $32-41$ & $29-38$ & $18-33$ \\
\cline { 2 - 7 } & 0.71 & $29-47$ & $36-48$ & $39-46$ & $38-42$ & $32-39$ & $21-35$ \\
\cline { 2 - 7 } & 1.00 & $30-57$ & $47-64$ & $46-59$ & $42-46$ & $36-46$ & $21-34$ \\
\hline
\end{tabular}

TABLE IV

Average Minimum Number of Iterations $n_{0}$ Such That $r\left(n_{0}\right)<$ $10^{-2}$ IS SATISFIED FOR DiFFERENT VALUES OF STANDARD DEVIATION $\sigma$ AND CORRELATION LENGTH $L_{c}$ AND FOR VV AND HH POLARIZATIONS. ANGLE OF INCIDENCE $=60^{\circ}$. SURFACE AUTOCORRELATION FUNCTION:

EXPONENTIAL. RElative Dielectric CONSTANT: $\epsilon_{r}=15-j 4$

\begin{tabular}{||c|c|c|c|c|c|c|c||}
\hline $\begin{array}{c}\text { Iterative } \\
\text { Method }\end{array}$ & $\begin{array}{c}\sigma / \lambda \\
\sigma / L_{c}\end{array}$ & $\begin{array}{c}0.1 \\
\text { VV-HH }\end{array}$ & $\begin{array}{c}0-3 \\
\text { VV-HH }\end{array}$ & $\begin{array}{c}0.5 \\
\text { VV-HH }\end{array}$ & $\begin{array}{c}0.7 \\
\text { VV-HH }\end{array}$ & $\begin{array}{c}1.0 \\
\text { VV-HH }\end{array}$ & $\begin{array}{c}10.0 \\
\text { VV-HH }\end{array}$ \\
\hline \multirow{3}{*}{ CGS } & 0.15 & $12-25$ & $13-27$ & $19-28$ & $17-26$ & $\mathbf{1 9 - 3 4}$ & nc-nc \\
\cline { 2 - 8 } & 0.20 & $13-23$ & $16-30$ & $19-31$ & $\mathbf{1 7 - 3 3}$ & $\mathbf{2 3 - 4 3}$ & nc-nc \\
\cline { 2 - 8 } & 0.33 & $13-22$ & $21-33$ & $\mathbf{2 2 - 3 3}$ & $\mathbf{2 7 - 4 5}$ & $\mathbf{3 1 - 4 9}$ & nc-nc \\
\cline { 2 - 8 } & 0.71 & $15-24$ & $\mathbf{2 3 - 3 3}$ & $\mathbf{3 2 - 4 5}$ & $\mathbf{4 2 - 4 9}$ & $\mathbf{4 1 - 5 5}$ & nc-nc \\
\cline { 2 - 8 } & 1.00 & $14-26$ & $\mathbf{2 4 - 3 6}$ & $\mathbf{5 2 - 5 1}$ & nc-nc & nc-nc & nc-nc \\
\hline Bi-CGSTAB & 0.15 & $17-34$ & $17-34$ & $21-34$ & $20-35$ & $\mathbf{3 3 - 3 5}$ & nc-nc \\
\cline { 2 - 8 } & 0.20 & $18-34$ & $22-38$ & $31-41$ & $\mathbf{2 6 - 4 4}$ & $\mathbf{4 2 - 4 4}$ & nc-nc \\
\cline { 2 - 8 } & 0.33 & $21-32$ & $27-39$ & $\mathbf{3 7 - 4 6}$ & $\mathbf{4 7 - 5 1}$ & $\mathbf{5 6 - 5 3}$ & nc-nc \\
\cline { 2 - 8 } & 0.71 & $23-28$ & $\mathbf{3 9 - 4 2}$ & $\mathbf{5 6 - 5 5}$ & $\mathbf{5 8 - 6 6}$ & $\mathbf{6 8 - 7 7}$ & nc-nc \\
\cline { 2 - 8 } & 1.00 & $24-29$ & $\mathbf{5 6 - 5 5}$ & $\mathbf{6 3 - 6 6}$ & nc-nc & nc-nc & nc-nc \\
\hline \multirow{5}{*}{ QMR } & 0.15 & $23-33$ & $28-36$ & $32-38$ & $35-56$ & $\mathbf{3 8 - 4 9}$ & nc-nc \\
\cline { 2 - 8 } & 0.20 & $25-36$ & $28-42$ & $34-44$ & $\mathbf{3 7 - 5 9}$ & $\mathbf{4 3 - 4 3}$ & nc-nc \\
\cline { 2 - 8 } & 0.33 & $25-34$ & $31-48$ & $\mathbf{4 0 - 5 4}$ & $\mathbf{4 1 - 7 8}$ & $\mathbf{5 4 - 6 5}$ & nc-nc \\
\cline { 2 - 7 } & 0.71 & $26-38$ & $\mathbf{4 2 - 6 0}$ & $\mathbf{5 4 - 5 8}$ & $\mathbf{7 4 - 8 8}$ & $\mathbf{9 6 - 9 8}$ & nc-nc \\
\cline { 2 - 7 } & 1.00 & $27-40$ & $\mathbf{4 5 - 7 9}$ & $\mathbf{7 2 - 7 8}$ & nc-nc & nc-nc & nc-nc \\
\hline
\end{tabular}

convergence rate than all the nonstationary methods when it converges. However, there are cases where FBM cannot converge while nonstationary algorithms can, in particular for the exponentially correlated surfaces as shown in Tables II and IV. Unfortunately, none of the methods converge for exponentially correlated surfaces when the surface is very rough (i.e., $\sigma / \lambda=10$ ). On the other hand, CGS has a surprisingly faster convergence rate among the nonstationary algorithms. Although in [24] CGS is reported to have highly irregular convergence behavior, it has converged consistently with a reasonable good convergence rate in all cases considered in this paper. Especially, for higher $\sigma / L_{c}$ ratios, $n_{0}$ values are significantly lower than the other nonstationary algorithms. Therefore, when electromagnetic wave scattering from 
(a)

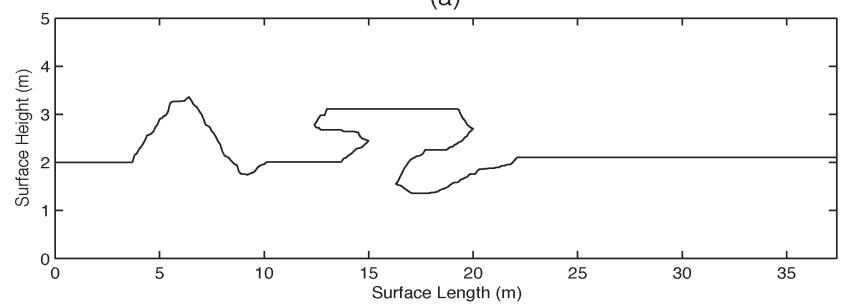

(b)

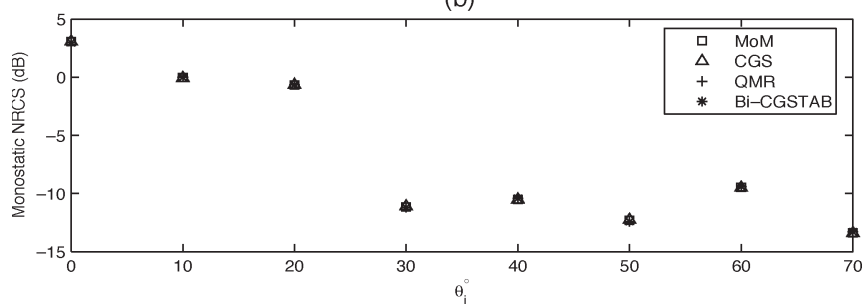

(c)

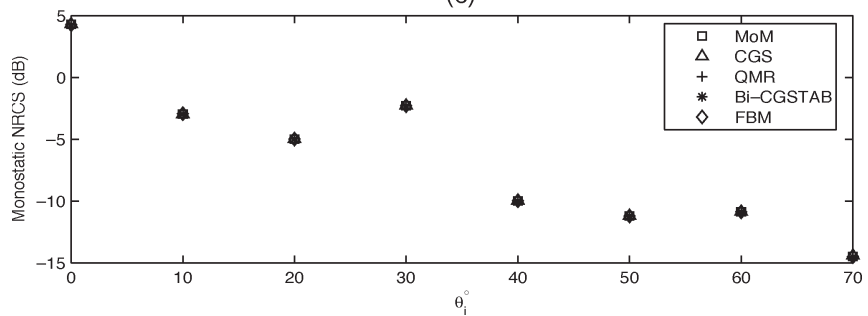

Fig. 5. (a) Geometry of a deterministic dielectric reentrant surface profile. (b) Comparison of monostatic NRCS values of MoM, CGS, QMR, and BiCGSTAB for the reentrant surface profile illustrated at (a) for $\mathrm{HH}$ polarization. Frequency is $300 \mathrm{MHz}$, and relative dielectric constant $\epsilon_{r}=15-j 4$. (c) Comparison of monostatic NRCS values of MoM, CGS, QMR, BiCGSTAB, and FBM for the reentrant surface profile illustrated at (a) for VV polarization. Frequency is $300 \mathrm{MHz}$, and $\epsilon_{r}=15-j 4$.

dielectric random rough surface profiles is investigated, CGS might be considered among the best candidates. One final observation is that $n_{0}$ values for VV polarization are generally less than the $n_{0}$ values for $\mathrm{HH}$ polarization. This is mainly due to the different coupled IEs used for $\mathrm{HH}$ and VV polarization cases. Magnetic field IE (MFIE) is used for VV polarization, whereas electric field IE (EFIE) is used for $\mathrm{HH}$ polarization and is known to exhibit poorer conditioning for interaction matrices when perfectly conducting and impedance rough surfaces have been considered [15]. Similarly, for dielectric rough surfaces, HH polarization examples yield more ill-conditioned systems than VV polarization cases for the same surface profiles.

\section{More General Cases and Advantages of Nonstationary Algorithms}

Finally, it has been shown that nonstationary algorithms are very suitable for more general dielectric rough surfaces. Therefore, a deterministic reentrant (i.e., multivalued) rough surface profile, which resembles a section of a natural terrain, is considered in Fig. 5(a) with $\epsilon_{r}=15-j 4$ and a frequency of $300 \mathrm{MHz}$. The monostatic NRCS values evaluated using iterative techniques are compared with the conventional MoM results for both $\mathrm{HH}$ and VV polarizations as illustrated in Fig. 5(b) and (c), respectively. Similar to the monostatic NRCS comparisons, bistatic NRCS comparisons have been performed for this geometry for both polarizations as shown in Fig. 6, where the incident angle is set to $75^{\circ}$. Finally, in Fig. 7, (a)

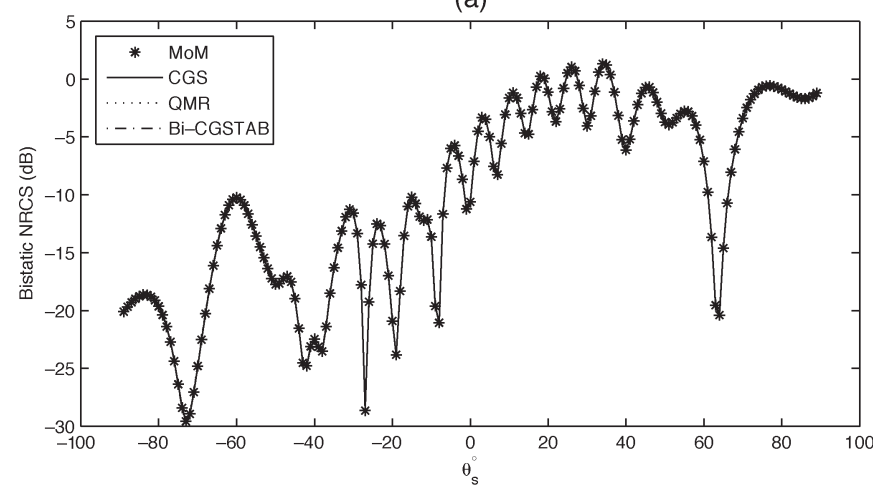

(b)

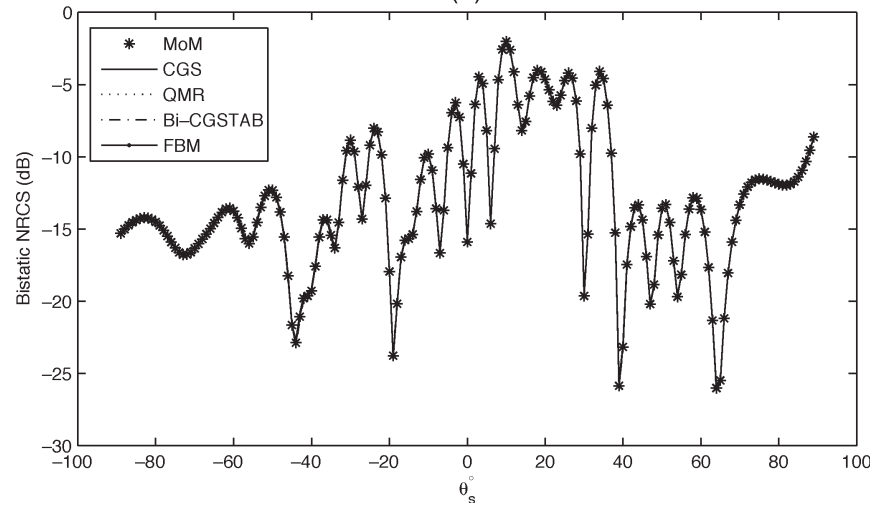

Fig. 6. Comparison of the bistatic NRCS values of the iterative methods with the numerically exact solution, MoM, for the reentrant profile given in Fig. 5(a). $\epsilon_{r}=15-j 4$, frequency $=300 \mathrm{MHz}$, and incident angle is set to $75^{\circ}$. (a) $\mathrm{HH}$ polarization. (b) $\mathrm{VV}$ polarization.

the magnitude of the surface electric current density $\left|\mathbf{J}_{s}\right|$, $\left[\hat{n} \times \mathbf{H}=\mathbf{J}_{s}\right.$ (in amperes per meter) $]$ and the magnitude of the total electric field $|\mathbf{E}|$ (in volts per meter) versus the surface length are obtained for the $\mathrm{HH}$ polarization case and compared with the conventional MoM results for the angle of incidence ranging from $0^{\circ}$ to $60^{\circ}$ with $20^{\circ}$ steps. A similar plot is given for the VV polarization in Fig. 8, where the iteratively obtained results for the magnitude of the surface magnetic current density $\left|\mathbf{K}_{s}\right|\left[\hat{n} \times \mathbf{E}=-\mathbf{K}_{s}\right.$ (in volts per meter) $]$ and the magnitude of the total magnetic field $|\mathbf{H}|$ (in amperes per meter) along the same surface length are compared with the conventional MoM results for the same angle of incidence values. In all results, excellent agreement is observed for both polarizations such that the rms error is less than $0.05 \mathrm{~dB}$. The convergence rate of each considered iterative method in terms of $n_{0}$ (i.e., minimum number of iterations) for different angles of incidence, when a predetermined error criterion $r\left(n_{0}\right)<0.01$ is satisfied, is tabulated in Table V. Although CGS and Bi-CGSTAB methods have similar convergence rates for the HH polarization, CGS method convergences significantly faster for the VV polarization. On the other hand, both CGS and Bi-CGSTAB methods perform better than the QMR method, in particular as the angle of incidence approaches to the grazing angle. An interesting result is the convergence of FBM for VV polarization, although the investigated surface is a reentrant one. FBM is known to have convergence problems for perfectly and imperfectly conducting reentrant surface profiles, where the IBC is used for the latter one. Besides the fact that for VV polarization MFIE is used, which is better suited for FBM, one way to explain its 


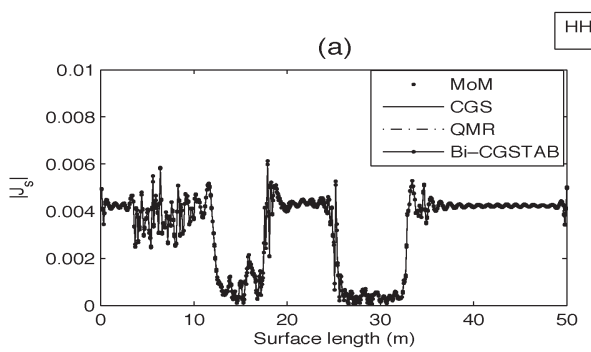

HH Polarization

(b)

(c)

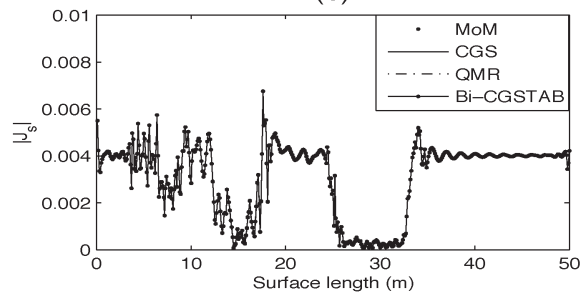

(d)
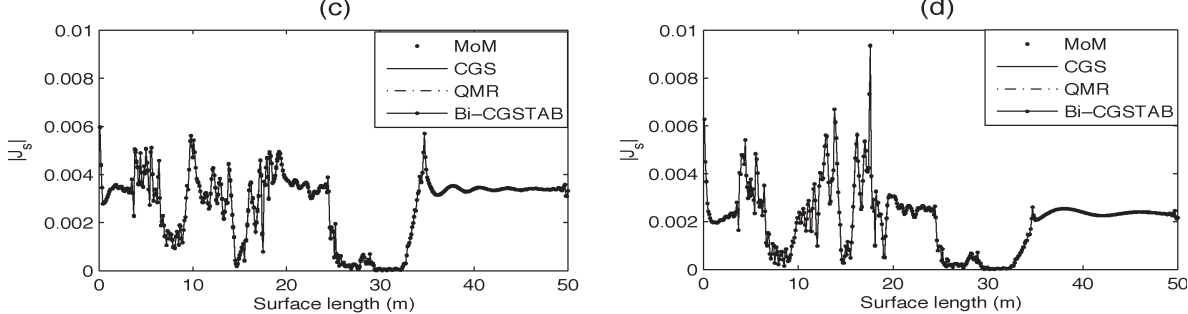

$(e)$

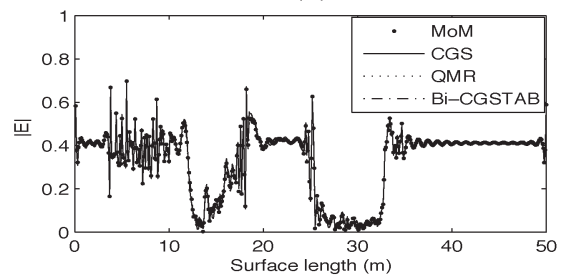

(f)

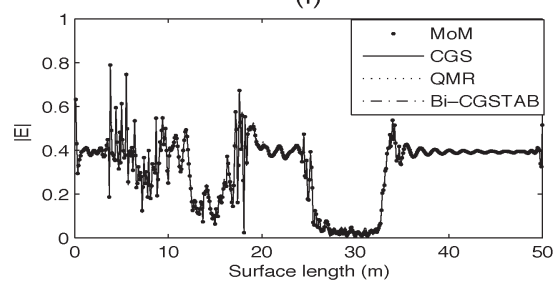

(g)

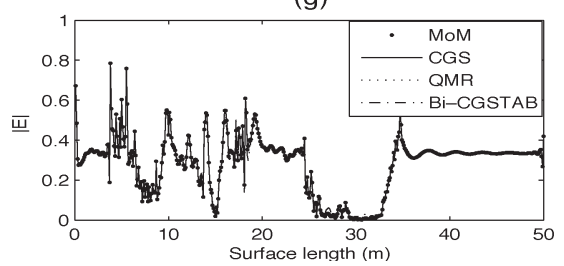

(h)

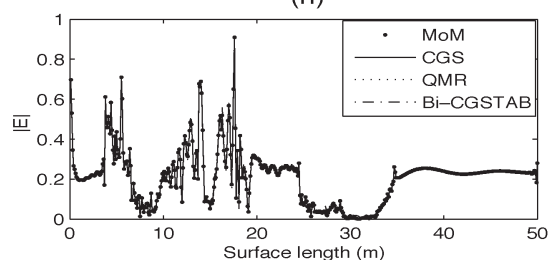

Fig. 7. Comparison of the magnitude of electric current distribution $\left|J_{s}\right|$ (in amperes per meter) and the total electric field $|E|$ (in volts per meter) for angles of incidence $0^{\circ}-60^{\circ}$ with $20^{\circ}$ steps calculated by MoM, CGS, QMR, and Bi-CGSTAB for HH polarization. The reentrant surface profile is the same as in Fig. 5(a). Frequency is $300 \mathrm{MHz}$, and $\epsilon_{r}=15-j 4$. (a) $\theta_{i}=0^{\circ}$. (b) $\theta_{i}=20^{\circ}$. (c) $\theta_{i}=40^{\circ}$. (d) $\theta_{i}=60^{\circ}$. (e) $\theta_{i}=0^{\circ}$. (f) $\theta_{i}=20^{\circ}$. (g) $\theta_{i}=40^{\circ}$. (h) $\theta_{i}=60^{\circ}$.

convergence for dielectric reentrant surfaces is to consider the cavity type issues that cause problems for FBM with reentrant surfaces. Essentially, cavities are hard to handle since they can contain lots of bounces. However, they are greatly reduced in the dielectric case. In particular if the dielectric constant is low and there is no conductivity, the bounces will die off quickly. This is easily verified by increasing the conductivity values of the complex dielectric constant. When conductivity is increased, the dielectric medium can be represented by an imperfectly conducting surface (and hence, corresponding IE is obtained using IBC). In the limiting case, where conductivity approaches to infinity, a perfectly conducting medium is achieved. As a result, when the conductivity is slightly increased, the convergence rate of FBM decreases, and finally, it does not converge at all. Furthermore, for the VV polarization, the Brewster effect may make the bounces die off quicker than in $\mathrm{HH}$ polarization.

\section{Conclusion}

Stationary and nonstationary iterative methods are applied to the problem of electromagnetic wave scattering from dielectric rough surfaces. Both random and deterministic surface profiles are considered. Accuracy, efficiency, and convergence issues of FBM as a stationary iterative method and CGS, QMR and Bi-CGSTAB as nonstationary iterative methods are investigated by comparing both the far-field and surface field results with those obtained from a conventional MoM solution for various random and deterministic surface profiles. Similar to the perfectly conducting and imperfectly conducting surface profiles, FBM has the quickest convergence rate if it converges. However, nonstationary methods are inherently more robust, and they converge for many different surface profiles where FBM fails to converge. Interestingly, among the nonstationary techniques, CGS appears to be the most suitable nonstationary algorithm for dielectric rough surface scattering calculations considered in this paper. On the other hand, unlike other nonstationary iterative methods, convergence problems have been observed for Bi-CGSTAB method for $\mathrm{HH}$ polarization when a Jacobi preconditioner is used. Thus, a block-diagonal preconditioner is used to obtain convergent results. Finally, as opposed to the perfectly and imperfectly conducting rough surface cases, FBM works fairly well for dielectric reentrant surface profiles for the VV polarization case. 


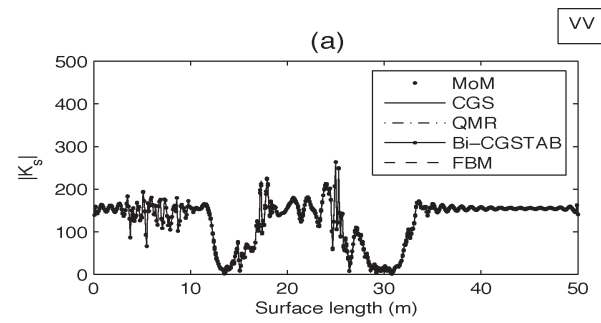

VV Polarization

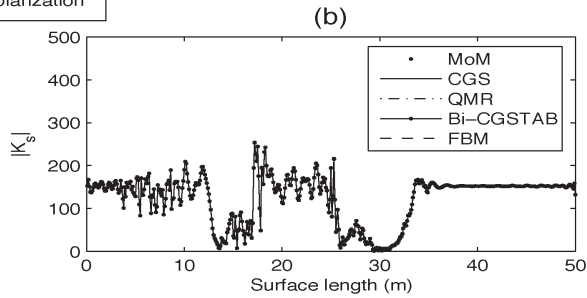

(c)
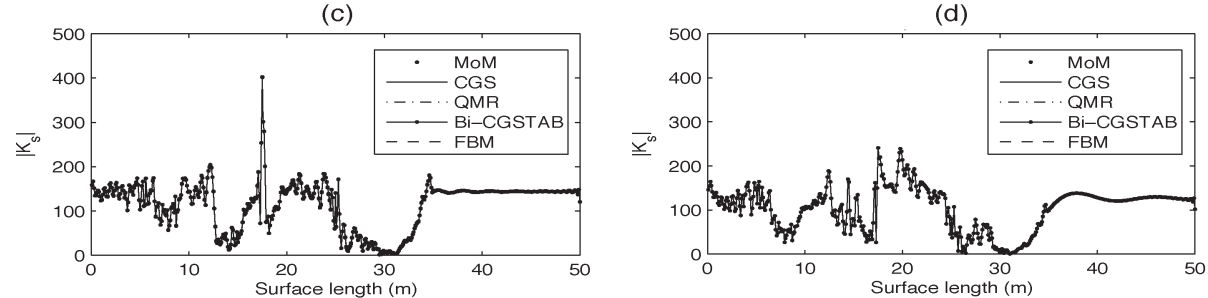

(e)

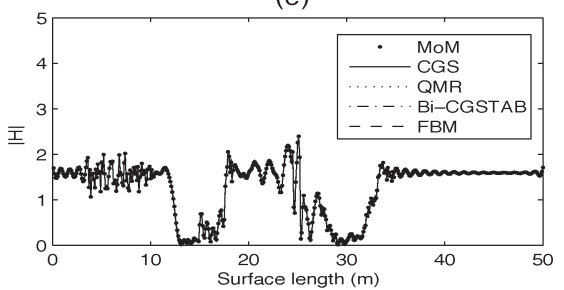

(f)

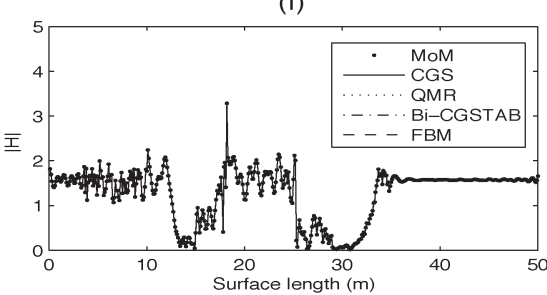

(g)
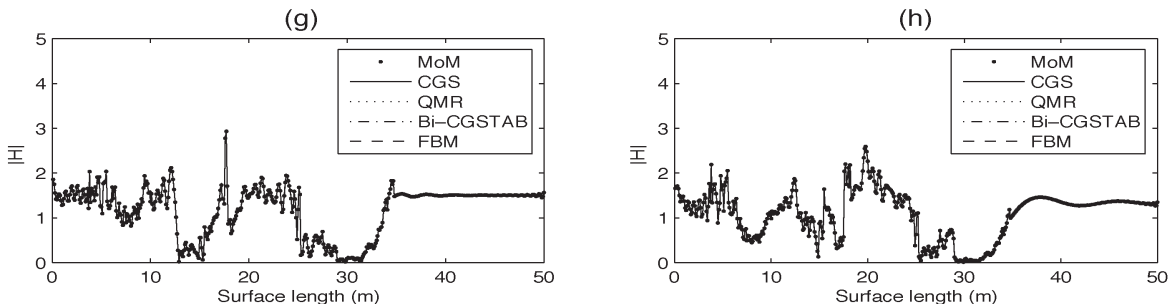

Fig. 8. Comparison of the magnitude of the magnetic current distribution $\left|K_{s}\right|$ (in volts per meter) and the total magnetic field $|H|$ (in amperes per meter) for angles of incidence $0^{\circ}-60^{\circ}$ with $20^{\circ}$ steps calculated by MoM, CGS, QMR, Bi-CGSTAB, and FBM for VV polarization. The reentrant surface profile is the same as in Fig. 5(a). Frequency is $300 \mathrm{MHz}$, and $\epsilon_{r}=15-j 4$. (a) $\theta_{i}=0^{\circ}$. (b) $\theta_{i}=20^{\circ}$. (c) $\theta_{i}=40^{\circ}$. (d) $\theta_{i}=60^{\circ}$. (e) $\theta_{i}=0^{\circ}$. (f) $\theta_{i}=20^{\circ}$. (g) $\theta_{i}=40^{\circ}$. (h) $\theta_{i}=60^{\circ}$.

\section{APPENDIX}

Full expressions of the coefficients of (6) are given [17], [21], i.e.,

$$
\begin{aligned}
S_{0 m n} & =\frac{1}{2} \delta_{m n}+\int_{\Delta l_{n}} \hat{n}^{\prime} \cdot \nabla \phi_{0} d l^{\prime} \\
& \cong\left\{\begin{array}{cc}
\frac{1}{2}-\frac{\left(d^{2} z / d x^{2}\right)_{n} \Delta x}{4 \pi\left[1+(d z / d x)_{n}^{2}\right]}, & m=n \\
\frac{j k_{0}}{4}\left(\hat{n}_{n} \cdot \mathbf{R}\right) H_{1}^{(2)}\left(k_{0}\left|\mathbf{r}_{m}-\mathbf{r}_{n}\right|\right) & \\
\times \sqrt{1+(d z / d x)_{n}^{2}} \Delta x, & m \neq n
\end{array}\right.
\end{aligned}
$$

$$
\begin{aligned}
Z_{0 m n} & =j \omega \mu_{0} \int_{\Delta l_{n}} \phi_{0} d l^{\prime} \\
& \cong j \omega \mu_{0}\left\{\begin{array}{cc}
-\frac{j}{4}\left(1-\frac{2 j}{\pi} \ln \frac{\gamma k_{0} \sqrt{1+(d z / d x)_{n}^{2}} \Delta x}{4 e}\right) & \\
\times \sqrt{1+(d z / d x)_{n}^{2}} \Delta x, & m=n \\
-\frac{j}{4} H_{0}^{(2)}\left(k_{0}\left|\mathbf{r}_{m}-\mathbf{r}_{n}\right|\right) & \\
\times \sqrt{1+(d z / d x)_{n}^{2}} \Delta x, & m \neq n
\end{array}\right.
\end{aligned}
$$

$$
\begin{aligned}
& S_{1 m n}=\frac{1}{2} \delta_{m n}-\int_{\Delta l_{n}} \hat{n}^{\prime} \cdot \nabla \phi_{1} d l^{\prime} \\
& \cong \begin{cases}\frac{1}{2}+\frac{\left(d^{2} z / d x^{2}\right)_{n} \Delta x}{4 \pi\left[1+(d z / d x)_{n}^{2}\right]}, & m=n \\
-\frac{j k_{1}}{4}\left(\hat{n}_{n} \cdot \mathbf{R}\right) H_{1}^{(2)}\left(k_{1}\left|\mathbf{r}_{m}-\mathbf{r}_{n}\right|\right) & \\
\quad \times \sqrt{1+(d z / d x)_{n}^{2}} \Delta x, & m \neq n\end{cases} \\
& Z_{1 m n}=-j \omega \mu_{0} \int_{\Delta l_{n}} \phi_{1} d l^{\prime} \\
& \cong j \omega \mu_{0}\left\{\begin{array}{ccc} 
& \frac{j}{4}\left(1-\frac{2 j}{\pi} \ln \frac{\gamma k_{1} \sqrt{1+(d z / d x)_{n}^{2}} \Delta x}{4 e}\right) & \\
& \times \sqrt{1+(d z / d x)_{n}^{2}} \Delta x, & \\
\frac{j}{4} H_{0}^{(2)}\left(k_{1}\left|\mathbf{r}_{m}-\mathbf{r}_{n}\right|\right) & \\
\times \sqrt{1+(d z / d x)_{n}^{2}} \Delta x, & m \neq n .
\end{array}\right.
\end{aligned}
$$

In (11)-(14), $\gamma=\exp (0.5772 \ldots)$, i.e., the exponential of Euler's constant, and $\mathbf{R}=\left(\mathbf{r}_{m}-\mathbf{r}_{n}\right) /\left|\mathbf{r}_{m}-\mathbf{r}_{n}\right|$, with $\mathbf{r}_{m}$ and $\mathbf{r}_{n}$ representing position vectors from the origin to the surface 
TABLE V

NUMBER OF ITERATIONS $n_{0}$ SUCH THAT $r\left(n_{0}\right)<0.01$ IS SATISFIED FOR DIFFERENT ANGLES OF INCIDENCE FOR DIELECTRIC REENTRANT SuRface Given in Fig. 5(a). Relative Dielectric Constant: $\epsilon_{r}=15-j 4$. FREQUENCY IS $300 \mathrm{MHz}$

\begin{tabular}{|c|c|c|c|c|}
\hline \multirow{2}{*}{$\begin{array}{c}\text { Incident Angle } \\
\text { (Degrees) }\end{array}$} & \multicolumn{4}{|c|}{ Iterative Method } \\
\cline { 2 - 5 } & GGS (VV-HH) & QMR (VV-HH) & Bi-CGSTAB (VV-HH) & FBM (VV-HH) \\
\hline 0 & $18-50$ & $40-48$ & $29-46$ & $12-\mathrm{nc}$ \\
\hline 10 & $21-36$ & $47-57$ & $30-48$ & $13-\mathrm{nc}$ \\
\hline 20 & $25-55$ & $51-99$ & $48-63$ & $17-\mathrm{nc}$ \\
\hline 30 & $27-56$ & $47-77$ & $45-56$ & $17-\mathrm{nc}$ \\
\hline 40 & $32-56$ & $46-98$ & $31-55$ & $17-\mathrm{nc}$ \\
\hline 50 & $23-63$ & $49-91$ & $28-42$ & $17-\mathrm{nc}$ \\
\hline 60 & $27-59$ & $53-109$ & $47-43$ & $17-\mathrm{nc}$ \\
\hline 70 & $20-61$ & $52-106$ & $38-58$ & $14-\mathrm{nc}$ \\
\hline
\end{tabular}

points at $x_{m}$ and $x_{n}$, respectively. Note that in (11) and (13), the second term for $m=n$ is small and, hence, can be ignored in the numerical computation. Furthermore, if a linear approximation of the surface contour is used, this term is zero. All of the equations for the $\mathrm{HH}$ polarization case, in view of the duality theorem, should be modified in a sense that electric fields and current densities must be replaced by magnetic ones. In addition, $\mu_{0}$ must be replaced by $\epsilon_{0}$ through $Z_{0 m n}$ calculation, and $\mu_{0}$ must be replaced by $\epsilon_{1}$ through $Z_{1 m n}$ calculation.

\section{REFERENCES}

[1] L. Tsang, J. A. Kong, and K.-H. Ding, Scattering of Electromagnetic Waves: Theories and Applications. New York: Wiley, 2000.

[2] L. Tsang, J. A. Kong, K. H. Ding, and C. O. Ao, Scattering of Electromagnetic Waves-Numerical Simulations. Hoboken, NJ: Wiley, 2001.

[3] J. C. West and J. M. Sturm, "On iterative approaches for electromagnetic rough-surface scattering problems," IEEE Trans. Antennas Propag., vol. 47, no. 8, pp. 1281-1288, Aug. 1999.

[4] R. M. Axline and A. K. Fung, "Numerical computation of scattering from a perfectly conducting random surface," IEEE Trans. Antennas Propag., vol. AP-26, no. 3, pp. 482-488, May 1978.

[5] A. K. Fung and M. F. Chen, "Numerical simulation of scattering from simple and composite random surfaces," J. Opt. Soc. Amer, vol. 2, no. 12, pp. 2274-2284, Dec. 1985.

[6] D. Holliday, L. L. DeRaad, and G. C. St-Cyr, "Forward-backward: A new method for computing low-grazing angle scattering," IEEE Trans. Antennas Propag., vol. 44, no. 5, pp. 722-729, May 1996.

[7] D. A. Kapp and G. S. Brown, "A new numerical method for rough surface scattering calculations," IEEE Trans. Antennas Propag., vol. 44, no. 5, pp. 711-721, May 1996.

[8] P. Tran, "Calculation of the scattering electromagnetic waves from a twodimensional perfectly conducting surface using the method of ordered multiple interactions," Waves Random Media, vol. 7, no. 3, pp. 295-302, Jul. 1997.

[9] C. F. Smith, A. F. Peterson, and R. Mittra, "The biconjugate gradient method for electromagnetic scattering," IEEE Trans. Antennas Propag., vol. 38, no. 6, pp. 938-940, Jun. 1990.

[10] D. J. Donohue, H. C. Ku, and D. R. Thompson, "Application of iterative moment-method solutions to ocean surface radar scattering," IEEE Trans. Antennas Propag., vol. 46, no. 1, pp. 121-132, Jan. 1998.

[11] F. Chen, "The numerical calculation of two-dimensional rough surface scattering by the conjugate gradient method," Int. J. Remote Sens., vol. 17, no. 4, pp. 801-808, 1996.

[12] M. R. Pino, L. Landesa, J. L. Rodrigues, F. Obellerio, and R. J. Burkholder, "The generalized forward-backward method for analyzing the scattering targets on ocean-like surfaces," IEEE Trans. Antennas Propag., vol. 47, no. 6, pp. 961-969, Jun. 1999.

[13] D. Torrungrueng and J. T. Johnson, "Numerical studies of back-scattering enhancement of electromagnetic waves from two-dimensional random rough surfaces using the FB/NSA method," J. Opt. Soc. Amer. A, Opt. Image Sci., vol. 18, no. 10, pp. 2518-2526, Oct. 2001.

[14] H. T. Chou and J. T. Johnson, "A novel acceleration algorithm for the computation of scattering from rough surfaces with the forward-backward method," Radio Sci., vol. 33, no. 5, pp. 1277-1287, Sep./Oct. 1998.
[15] J. C. West, "Integral equation formulation for iterative calculation of scattering from lossy rough surfaces," IEEE Trans. Geosci. Remote Sens., vol. 38, no. 4, pp. 1609-1615, Jul. 2000.

[16] B. Babaoglu, A. Altintas, and V. B. Erturk, "Spectrally accelerated biconjugate gradient stabilized method for scattering from and propagation over electrically large inhomogeneous geometries," Microw. Opt. Technol. Lett., vol. 46, no. 2, pp. 158-162, Jul. 2005.

[17] M. F. Chen and S. Y. Bai, "Computer simulation of wave scattering from a dielectric random surface in two dimensions-Cylindrical case," J. Electromagn. Waves Appl., vol. 4, no. 10, pp. 963-982, 1990.

[18] A. Benali, J. Chandezon, and J. Fontaine, "A new theory for scattering of electromagnetic waves from conducting or dielectric rough surfaces," IEEE Trans. Antennas Propag., vol. 40, no. 2, pp. 141-148, Feb. 1992.

[19] K. Sarabandi, Y. Oh, and F. T. Ulaby, "A numerical simulation of scattering from one-dimensional inhomogeneous dielectric random surfaces," IEEE Trans. Geosci. Remote Sens., vol. 34, no. 2, pp. 425-432, Mar. 1996.

[20] Q. Li, C. H. Chan, and L. Tsang, "Monte Carlo simulations of wave scattering from lossy dielectric random rough surfaces using the physicsbased two-grid method and the canonical-grid method," IEEE Trans. Antennas Propag., vol. 47, no. 4, pp. 752-763, Apr. 1999.

[21] G. Franceschetti, A. Iodice, and D. Riccio, "Scattering from dielectric random fractal surfaces via method of moments," IEEE Trans. Geosci. Remote Sens., vol. 38, no. 4, pp. 1644-1655, Jul. 2000.

[22] A. Iodice, "Forward-backward method for scattering from dielectric rough surfaces," IEEE Trans. Antennas Propag., vol. 50, no. 7, pp. 901-911, Jul. 2002.

[23] _ , "Scattering from natural soils modeled by dielectric fractal profiles: The forward-backward approach," IEEE Trans. Geosci. Remote Sens. vol. 42, no. 1, pp. 77-85, Jan. 2004.

[24] R. Barrett, M. Berry, T. F. Chan, J. Demmel, J. M. Donato, J. Dongarra, V. Eijkhout, R. Pozo, C. Romine, and H. Vorst, Templates for the Solution of Linear Systems: Building Blocks for Iterative Methods. Philadelphia, PA: SIAM, 1994

[25] X. S. Lee, "Use of parallel SuperLU in large scale scientific calculations," in Proc. SIAM Annu. Meet., Portland, OR, Jul. 12-16, 2004.

[26] M. F. Chen and A. K. Fung, "A numerical study of the regions of validity of the Kirchoff and small perturbation rough surface scattering models," Radio Sci., vol. 23, no. 2, pp. 163-170, 1988.

[27] R. Mittra and A. Peterson, Numerical Techniques for RCS Computation and Scattering Center Approach to RCS Modeling. Lecture Note, vol. 2.

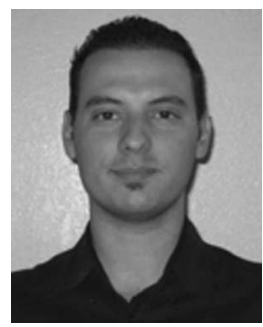

K. Inan received the B.S. and M.S. degrees from Bilkent University, Ankara, Turkey, in 2002 and 2005, respectively.

Since 2005, he has been a Research Assistant with the Electronic Packaging Laboratory of the Electrical Engineering Department, Arizona State University, Mesa. His research interests include rough surface scattering, computational electromagnetics, and high-speed electronics packaging.

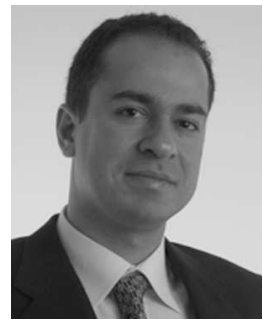

V. B. Ertürk (M'99) received the B.S. degree in electrical engineering from the Middle East Technical University, Ankara, Turkey, in 1993, and the M.S. and Ph.D. degrees from The Ohio State University, Columbus, in 1996 and 2000, respectively.

$\mathrm{He}$ is currently an Assistant Professor with the Electrical and Electronics Engineering Department, Bilkent University, Ankara. His research interests include the analysis and design of planar and conformal arrays, active integrated antennas, scattering from and propagation over large terrain profiles, as

well as metamaterials.

Dr. Ertürk has served as the Secretary/Treasurer of the IEEE Turkey Section as well as the Turkey Chapter of the IEEE Antennas and Propagation, Microwave Theory and Techniques, Electron Devices, and Electromagnetic Compatibility Societies. 